OPEN ACCESS

Edited by:

Oliver Wirths,

Universitätsmedizin Göttingen,

Germany

Reviewed by:

Sara Baldelli,

San Raffaele University of Rome, Italy Melissa Calegaro Nassif,

Universidad Mayor, Chile

*Correspondence: Honglin Luo honglin.luo@hli.ubc.ca

Received: 05 January 2018 Accepted: 14 February 2018

Published: 12 March 2018

Citation: Xue YC, Feuer R, Cashman N and Luo H (2018) Enteroviral Infection: The Forgotten Link to Amyotrophic Lateral Sclerosis?

Front. Mol. Neurosci. 11:63. doi: 10.3389/fnmol.2018.00063

\section{Enteroviral Infection: The Forgotten Link to Amyotrophic Lateral Sclerosis?}

\author{
Yuan Chao Xue ${ }^{1,2}$, Ralph Feuer ${ }^{3}$, Neil Cashman ${ }^{4}$ and Honglin Luo ${ }^{1,2 *}$ \\ ${ }^{1}$ Centre for Heart and Lung Innovation, University of British Columbia, Vancouver, BC, Canada, ${ }^{2}$ Department of Pathology \\ and Laboratory Medicine, University of British Columbia, Vancouver, BC, Canada, ${ }^{3}$ The Integrated Regenerative Research \\ Institute at San Diego State University, San Diego, CA, United States, ${ }^{4}$ Djavad Mowafaghian Centre for Brain Health, \\ University of British Columbia, Vancouver, BC, Canada
}

Amyotrophic lateral sclerosis (ALS) is a devastating neurodegenerative disease that primarily attacks motor neurons in the brain and spinal cord, leading to progressive paralysis and ultimately death. Currently there is no effective therapy. The majority of ALS cases are sporadic, with no known family history; unfortunately the etiology remains largely unknown. Contribution of Enteroviruses (EVs), a family of positivestranded RNA viruses including poliovirus, coxsackievirus, echovirus, enterovirus-A71 and enterovirus-D68, to the development of ALS has been suspected as they can target motor neurons, and patients with prior poliomyelitis show a higher risk of motor neuron disease. Multiple efforts have been made to detect enteroviral genome in ALS patient tissues over the past two decades; however the clinical data are controversial and a causal relationship has not yet been established. Recent evidence from in vitro and animal studies suggests that enterovirus-induced pathology remarkably resembles the cellular and molecular phenotype of ALS, indicating a possible link between enteroviral infection and ALS pathogenesis. In this review, we summarize the nature of enteroviral infection, including route of infection, cells targeted, and viral persistence within the central nervous system (CNS). We review the molecular mechanisms underlying viral infection and highlight the similarity between viral pathogenesis and the molecular and pathological features of ALS, and finally, discuss the potential role of enteroviral infection in frontotemporal dementia (FTD), a disease that shares common clinical, genetic, and pathological features with ALS, and the significance of anti-viral therapy as an option for the treatment of ALS.

Keywords: amyotrophic lateral sclerosis, enterovirus, TDP-43 pathology, nucleocytoplasmic trafficking, RNA metabolism, autophagy, neuroinflammation

\section{INTRODUCTION}

Amyotrophic lateral sclerosis (ALS) is a fatal neurodegenerative disease marked by progressive degeneration of both upper and lower motor neurons, resulting in paralysis and eventual death within 3-5 years after diagnosis (Brown and Al-Chalabi, 2017; Hardiman et al., 2017; van Es et al., 2017). Despite primarily a motor neuron disease, nearly 50\% of ALS patients show cognitive/behavioral impairments, suggesting an involvement of non-motor systems in ALS pathogenesis (Hardiman et al., 2017). The overall prevalence of ALS in Europe and North America is estimated at $\sim 3-5$ cases per 100,000 people and increases with age (Brown and Al-Chalabi, 2017). 
The average age of ALS onset is between 55 and 65, with the cumulative lifetime risk being higher for men (1:350) than for women (1:400; van Es et al., 2017). There is currently no cure for ALS. Riluzole (a glutamate release inhibitor) and edaravone (a free-radical scavenger) are the only two FDA-approved drugs for the treatment of ALS. However, their benefits are modest by only delaying disease progression and prolonging survival for 2-3 months (Martinez et al., 2017). Many other therapeutic options have been investigated over the past two decades, but their clinical effectiveness has not been proven (Katyal and Govindarajan, 2017; Martinez et al., 2017).

ALS can be genetically inherited or occur sporadically in individuals without any apparent family history. Since the identification of SOD1 as a causative gene of ALS, significant progress has been made in unravelling the genetics causes of ALS. Over the past two decades, $\sim 30$ genes have been identified to be highly associated with ALS (Al-Chalabi et al., 2017; Ito et al., 2017; Chia et al., 2018). These genes encode proteins that are involved in the maintenance of protein homeostasis and vesicle trafficking (e.g., SQSTM1/p62, TBK1, SOD1, VCP, CHMP2B, OPTN, UBQLN2 and C9ORF72), RNA processing (e.g., TARDBP, FUS, TIA1, HNRNPA1, C9ORF72 and MATR3), and regulation of cytoskeletal integrity and axonal transport (e.g., PFN1, DCTN1, SOD1, and TUBA4A, Al-Chalabi et al., 2017; Mackenzie et al., 2017; Chia et al., 2018). Unlike familial ALS (FALS), the cause(s) of sporadic ALS (SALS), which accounts for the majority of ALS cases (90\%-95\%), remain(s) largely unclear. A "multistep model" of gene-environment interaction requiring both genetic mutations and environmental risk factors has been proposed as a mechanism triggering the onset and progression of SALS (Al-Chalabi et al., 2014). Several environmental risk factors have been studied, including viral exposure, physical activity, smoking, heavy metals, pesticides and chemicals, military service, and electric shock ( $\mathrm{Yu}$ and Pamphlett, 2017). However, a definitive relationship between these factors and ALS has yet to be established. This review will focus on the possible contribution of viral infection, in particular, enteroviral infection in the pathogenesis of ALS.

\section{ENTEROVIRUSES AND NEUROLOGICAL DISORDERS}

Enteroviruses (EVs) are a group of single, positive-stranded RNA viruses of the Picornaviridae family that include poliovirus, coxsackievirus, echovirus and enterovirus, with the latter (specifically EV-A71 and EV-D68) emerging as the causative agents of the recent large epidemics across the Asia-Pacific and North American region, respectively (Huang and Shih, 2015; Anastasina et al., 2017). Although EVs commonly cause asymptomatic infection, sometimes they are associated with severe diseases, including neurological complications. EVs have a high tropism for the central nervous system (CNS) and account for various neurological disorders, such as poliomyelitis, aseptic meningitis, encephalitis and non-polio flaccid paralysis, particularly in infants and children (Rhoades et al., 2011; Huang and Shih, 2015). Since the successful campaign of the poliovirus vaccination, neurological diseases caused by non-polio EVs have been increasingly reported. For example, acute flaccid paralysis was frequently observed among patients with EV-A71, echovirus and coxsackievirus infection (Suresh et al., 2018). In addition, epidemiological studies from the recent EV-D68 outbreaks reveal a strong relationship between EV-D68 infection and increased incidence of acute flaccid myelitis (Greninger et al., 2015; Messacar et al., 2016).

While the majority of the EVs are transmitted through the fecal-oral route and replicate in the gastrointestinal tract, some EVs (e.g., EV-D68) can cause respiratory infection and spread via respiratory secretion. Available evidence suggests that EVs can invade the CNS from these primary infection sites through three main mechanisms: (1) retrograde axonal transport-both poliovirus and EV-A71 can infect the peripheral nerve and gain access into the CNS via retrograde axonal transport and trans-synaptic spread (Gromeier and Wimmer, 1998; Chen et al., 2007); (2) blood-brain barrier (BBB) penetration-during viremia, poliovirus in the blood can directly cross the $\mathrm{BBB}$ through disrupted tight junctions that are likely induced by inflammation independently of viral receptor (Yang et al., 1997), and/or via transferrin receptor 1-mediated direct transmission (Mizutani et al., 2016); and (3) "Trojan-horse" invasion-EVs, such as poliovirus (Freistadt and Eberle, 1996), EV-A71 (Lin et al., 2009b) and coxsackievirus (Tabor-Godwin et al., 2010), can also invade the CNS through virus-infected immune cells, including macrophage/monocytes, dendritic cells, lymphocytes and nesting ${ }^{+}$myeloid cells, which act as carriers to deliver virus into the CNS. EVs likely utilize one or multiple routes of entry into the CNS.

Within the CNS, many cell types can be targeted by the EVs. It is well documented that poliovirus infects and replicates in motor neurons within the anterior horns of the spinal cord, leading to poliomyelitis (Nagata et al., 2004). Motor neurons in the spinal cord and brainstem are also highly susceptible to EV-71 (Ong and Wong, 2015; Too et al., 2016). More recently, it was reported that mice infected with EV-D68 isolates from the 2014 outbreak develop limb paralysis, closely resembling human acute flaccid myelitis, due to infection of motor neurons in the anterior horns of spinal cord (Hixon et al., 2017). In addition to neuronal cells, especially motor neurons, astrocytes and oligodendrocytes are also permissive to poliovirus (Couderc et al., 2002), EV-A71 (Tung et al., 2010), and coxsackievirus B3 (CVB3; Zhang et al., 2013). Moreover, CVB3 and EV-A71 were found to preferentially target neural progenitor cells compared with differentiated neuronal cells, suggesting a mechanism of viral persistence and possible lasting neurological consequences (Feuer et al., 2005; Tsueng et al., 2011; Huang et al., 2014).

Although EVs are regarded as highly lytic viruses and EV-related diseases are commonly resulted from acute infection, EVs, such as poliovirus (Julien et al., 1999), EV-A71 (Han et al., 2010), and coxsackievirus (Feuer et al., 2009), can persist in various tissues, including the CNS. Glial cells and neuronal progenitor cells were reported to be the sites of CVB3 persistence (Feuer et al., 2009; Zhang et al., 2013). Multiple viral and host factors, including viral receptors, viral 
mutations, viral evasion of host immune response, and host translation machinery, participate in establishing a persistent EV infection (Rhoades et al., 2011; Huang and Shih, 2015). Latent EVs might be reactivated years later, either spontaneously or in response to exogenous stimulations, such as local trauma (Andréoletti et al., 2000; Feuer et al., 2002). EV persistence in cardiomyocytes and pancreatic cells has been associated with chronic clinical conditions, such as dilated cardiomyopathy and type 1 diabetes, mainly through continuous inflammatory responses (Chapman and Kim, 2008; Oikarinen et al., 2012). However, the long-term impacts of EV infection within the CNS are largely unclear. Clinically, it is observed that polio survivors decades after the recovery from the acute paralytic poliomyelitis can develop post-poliomyelitis syndrome, a neurological disorder characterized by new and progressive muscular weakness, accompanied by the detection of defective viral particles in the cerebrospinal fluid of some patients (Dalakas, 1995), indicating a possible long-lasting effect of latent poliovirus infection. In addition, a murine model has shown that a neonatal CVB3 infection can have a chronic impact on neurogenesis and CNS development, further supporting a potential link between early subclinical infections and late neurological sequelae (Ruller et al., 2012).

\section{VIRAL INFECTION AND ALS}

Viral infection has long been suspected as an environmental risk factor and/or a causative pathogen for ALS. Over the past 30 years, many efforts have been made to explore the association of neurotropic viruses (Limongi and Baldelli, 2016), especially EVs, exogenous retroviruses such as human immunodeficiency virus (Verma and Berger, 2006) and human T cell leukemia virus (Araujo, 2015), and human endogenous retrovirus (HERV; Li et al., 2015), with ALS.

HERVs are the remnants of ancient retroviruses integrated into the human genome and normally inactivate, but can be re-activated under physiological and pathological stresses (Li et al., 2015). Despite unsuccessful attempts to find evidence of exogenous retroviruses in ALS patient tissues (McCormick et al., 2008), several studies have reported the detection of enhanced gene expression of HERV-K and reverse transcriptase activity in the blood (Andrews et al., 2000; Steele et al., 2005; MacGowan et al., 2007) and brain tissues (Douville et al., 2011; Li et al., 2015) of ALS patients. More notably, transgenic mice expressing HERV-K in the neurons develop progressive motor neuron dysfunction similar to human ALS phenotype (Li et al., 2015), suggesting a potential viral etiology of ALS. Further studies are underway to explore the mechanism by which HERV-K causes neuropathology and to test existing anti-retroviral drugs and develop novel anti-HERV inhibitors for the treatment of retrovirus-associated ALS (Bowen et al., 2016; Tyagi et al., 2017).

A potential role of EVs in ALS has been proposed for decades due to their ability to target motor neurons and the development of the ALS-like post-poliomyelitis-syndrome (Ravits, 2005). Multiple clinical studies have been conducted to detect EVs in ALS patient tissues; however, the available data are controversial and inconclusive. Using RT-PCR, three studies reported a $60 \%-88 \%$ incidence of EV genome detection in spinal cord/brain of ALS patients, compared to $0 \%-14 \%$ in controls (Woodall et al., 1994; Berger et al., 2000; Giraud et al., 2001). Additionally, RT-PCR analysis of cerebrospinal fluid showed EV detection in $14.5 \%$ of 242 ALS patients and $7.6 \%$ of 354 controls (Vandenberghe et al., 2010). However, three additional studies failed to detect EV RNA in spinal cord/brain of either ALS patients or controls (Swanson et al., 1995; Walker et al., 2001; Nix et al., 2004). The discrepancies between these studies are likely due to methodological differences, such as the use of fresh vs. archived (or frozen vs. fixed) tissues, and differences in PCR primers/amplification methods, which can all affect the integrity of viral RNA and the sensitivity/specificity of viral genome detection. Moreover, the stage of the disease when samples are collected may also be critical for viral detection, as viruses may be detectable or active only in certain phases of the disease or in only a subset of patients. In addition, given the emerging "prion-like mechanism" in ALS pathogenesis (Grad et al., 2015), it is also possible that $\mathrm{EV}$ infection causes disease pathology (i.e., seeding of protein misfolding) focally during the acute phase of childhood infection, followed by the gradual propagation of misfolded proteins in other regions of the CNS, eventually leading to ALS onset in adulthood. In such case, an active viral infection may not be required for disease progression. Studies involved populationbased retrospective cohort may be one way to "enrich" the chances of linking EV infection to later ALS. But the most common symptoms of EV infections are flu-like symptoms, which are often neglected until more disastrous consequences arise. Thus, the existing clinical EV infection data may not be an accurate reflection of the real infection prevalence. Overall, with these limitations, it would be very difficult to come up with a compelling strategy to firmly establish the connection between EV infection and ALS in humans. However, recent evidence from in vitro and animal studies prompts us to re-visit the longstanding controversial role of EVs in ALS from a different perspective.

\section{EV-INDUCED MOLECULAR AND PATHOLOGICAL CHANGES ASSOCIATED WITH ALS PATHOGENESIS}

Emerging evidence from cell culture and mouse experiments reveals that $\mathrm{EV}$ infection produces hallmark cellular and molecular phenotypes of ALS, including RNA-processing defects, impaired nucleocytoplasmic transport, neuroinflammation, compromised protein quality control, and most strikingly, TDP-43 (transactive response DNA binding protein-43) pathology, supporting a potential link between EV infection and ALS pathogenesis (Figure 1).

\section{RNA-Processing Defects and TDP-43 Pathology}

The significance of aberrant RNA metabolism in ALS is increasingly recognized since the discoveries of ALS-causing 


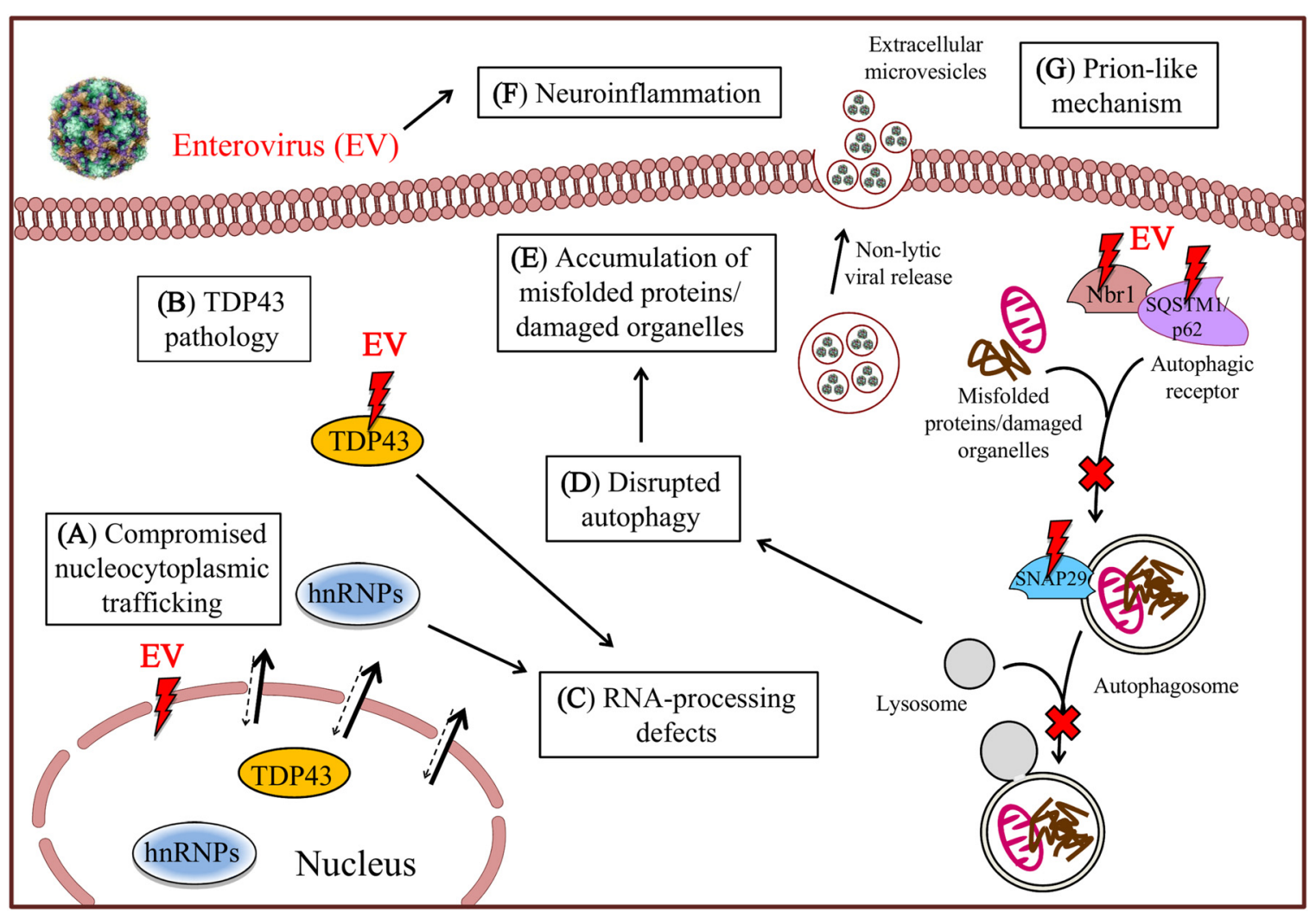

FIGURE 1 | Molecular and pathological similarities between amyotrophic lateral sclerosis (ALS) and enteroviral infection. Enterovirus (EV) infection impairs nucleocytoplasmic trafficking (A) via EV proteinase-mediated proteolysis of nucleoporin proteins, resulting in mislocalization of TDP-43 and heterogeneous nuclear ribonucleoproteins (hnRNPs) from the nucleus to the cytoplasm, where they are further cleaved to induce TDP-43 pathology (B) and cause RNA-processing defects (C). EV infection also results in the cleavage of several critical autophagic proteins, including SQSTM1/p62, Nbr1 and SNAP29, contributing to the disruption of the autophagic pathway (D) and consequent accumulation of misfolded proteins/damaged organelles (E). Finally, subclinical EV infection induces chronic inflammatory reaction (F) and promotes non-lytic viral spread and prion-like mechanism through extracellular microvesicles (G).

mutations in many genes that encode RNA-binding proteins (RBPs), including TARDBP, FUS, HNRNPA1, MATR3, TAF15 and TIA1 (Ito et al., 2017). More notably, cytoplasmic mislocalization, aggregation, and cleavage of TDP-43 (TDP-43 pathology) in motor neurons and glial cells have been found in $\sim 97 \%$ of all ALS cases, and consequently regarded as a pathological hallmark of ALS (Neumann et al., 2006; Mackenzie et al., 2007). TDP-43, encoded by TARDBP, is a ubiquitously expressed DNA/RNA-binding protein and plays a critical role in regulating RNA metabolism (Lee et al., 2012). Mechanistically, both loss- and gain-of-function in TDP-43 pathology are believed to contribute to disease onset and progression (Lee et al., 2012). The replication of EVs, including poliovirus, EV-A71, and coxsackievirus, which takes place in the cytoplasm, relies heavily on the function of cellular proteins. Numerous RBPs, which are mostly nucleocytoplasmic shuttling proteins and localized predominantly to the nucleus, are detained in the cytoplasm during EV infection, and hijacked by EVs for support of viral translation and viral RNA replication (Lloyd, 2015). For example, EV-A71 infection causes redistribution of heterogeneous nuclear ribonucleoprotein (hnRNP) Al from the nucleus to the cytoplasm, where it stimulates EV-A71 replication by facilitating internal ribosome entry site-mediated viral translation (Lin J. Y. et al., 2009; Tolbert et al., 2017). Most intriguingly, it was found that CVB3 infection results in cytoplasmic mislocalization and cleavage of TDP-43 via the action of CVB3-encoded proteinases, leading to RNA-processing deficits (Fung et al., 2015). Together, these findings suggest that EV infection shares a strikingly similar molecular feature in RNA metabolism with ALS.

\section{Compromised Nucleocytoplasmic Trafficking}

Defects in nucleocytoplasmic shuttling have recently been identified as a central theme of ALS research, contributing significantly to the pathological hallmark of cytoplasmic mislocalization of RBPs (Boeynaems et al., 2016). Although the underlying mechanisms remain largely elusive, recent studies demonstrate that the G4C2 hexanucleotide repeat expansion mutation in the first intron of chromosome 9 open reading frame 72 (C9orf72) plays an important role in the dysfunction of nucleocytoplasmic transport (Freibaum et al., 2015; Jovicic et al., 2015; Xiao et al., 2015; Zhang et al., 2015). Mutation in C9orf72 
is the most common genetic cause of ALS, responsible for $\sim 40 \%$ of FALS and $5 \%-10 \%$ of SALS (DeJesus-Hernandez et al., 2011; Renton et al., 2011). It was discovered that expression of $\mathrm{G} 4 \mathrm{C} 2$ repeats impairs nucleocytoplasmic transport at the levels of transcribed G4C2 repeat RNA and translated dipeptide repeat proteins through interfering with the function of Ran GTPase-activating protein 1 and by disrupting the integrity of the nuclear pore complex (NPC; Freibaum et al., 2015; Jovicic et al., 2015; Xiao et al., 2015; Zhang et al., 2015). Likewise, compromised nucleocytoplasmic trafficking is a common mechanism for EV-induced pathology (Yarbrough et al., 2014). It was found that nucleoporin (Nup) proteins, Nup62, Nup98 and Nup153, key components of the NPC, are targeted by poliovirus-encoded proteinase $2 \mathrm{~A}$ for degradation, resulting in the blockage of nuclear import (Gustin and Sarnow, 2001; Park et al., 2008, 2015). Through this strategy, EVs gain access to the otherwise predominantly nuclear proteins necessary for effective viral replication and inhibit host immune response by preventing nuclear transport of anti-viral signal molecules (Yarbrough et al., 2014). A recent finding that expression of $\mathrm{CVB} 3$ proteinase $2 \mathrm{~A}$ alone is sufficient to induce cytoplasmic accumulation of TDP-43 indicates a possible overlapping mechanism linking EV to ALS (Fung et al., 2015).

\section{Neuroinflammation}

The immune involvement in the development of ALS has been widely studied, with most focused on the activation of glial cells and astrocytes as such event would lead to the up-regulation of multiple pro-inflammatory cytokines, such as tumor necrosis factor- $\alpha$, monocyte chemoattractant protein-1, cyclooxygenase-2, and interleukins (Sekizawa et al., 1998; Almer et al., 2001; Robertson et al., 2001). For viral infection, beyond direct damage, EVs could also stimulate immune-mediated injury by facilitating the production of pro-inflammatory cytokines, leukocyte infiltration and astrogliosis (Lin et al., 2003; Feuer et al., 2009; Rhoades et al., 2011; Ruller et al., 2012). It was shown that chronic CVB3 infection induces CNS microgliosis and astrogliosis in persistently infected mouse brains (Feuer et al., 2009). The presence of immune cells and cytokines within the CNS can worsen the virus-mediated neuropathology and the potential bystander damage caused by the subsequent $\mathrm{T}$ cell activation (Lin et al., 2009a). Furthermore, neuroinflammation initiated by microglia and astrocytes can trigger cell death by promoting the production of reactive oxygen species. Altogether, current evidence supports the possibility that chronic EV infection is able to induce late-onset CNS dysfunction by inducing inflammatory reactions.

\section{Defective Autophagy}

In addition to RNA metabolism, ALS-causing mutations in genes, such as SQSTM1/p62, OPTN, TBK1, VCP and UBQLN2, are frequently related to protein quality control (Al-Chalabi et al., 2017; Chia et al., 2018). Autophagy deficits and consequent accumulation of misfolded proteins and large RNA clusters (e.g., RNA granules) are implicated in ALS pathogenesis (Cipolat Mis et al., 2016). Recent studies indicate that EVs can hijack the autophagic pathway to their own advantage. Autophagy is a dynamic process comprised of autophagosome formation and degradation following fusion with lysosome, called autophagic flux. Autophagic flux is inhibited upon $\mathrm{EV}$ infection, due at least in part to $\mathrm{EV}$ proteinase-mediated cleavage of synaptosomal-associated protein 29, a critical component of the soluble $\mathrm{N}$-ethylmaleimide-sensitive factor activating protein receptor (SNARE) complex required for autophagosome-lysosome fusion (Corona et al., in press; Mohamud et al., in press). As a result of reduced autophagic flux, autophagosomes accumulate and favor viral growth by providing membrane scaffolds for viral assembly and replication (Shi and Luo, 2012). Inhibition of autophagic flux also leads to accumulation of protein aggregates, contributing to viral pathogenesis. Apart from bulk degradation, autophagy can selectively recycle misfolded proteins/damaged organelles, a process mediated by autophagic receptors, including sequestosome 1 (SQSTM1)/p62 and neighbor of BRCA1 (Nbr1), which target ubiquitinated proteins/organelles to autophagosomes for destruction (Shaid et al., 2013). EV infection leads to cleavage of SQSTM1 and/or Nbr1 via the proteolytic activity of EV proteinases, resulting in impaired clearance of protein aggregates (Shi et al., 2013, 2014). Collectively, current evidence suggests a novel molecular mechanism employed by EVs to promote viral replication and induce ALS-like viral pathogenesis.

\section{Prion-Like Mechanism}

ALS is known to start focally and spread to other regions in a neuroanatomic fashion (Grad et al., 2015). Recent evidence suggests that both exosome-dependent and -independent mechanisms are involved in the transmission of misfolded proteins (Grad et al., 2014a,b). As non-enveloped viruses, EVs are traditionally suggested to only be able to exit the infected cell by causing it to rupture. However, it has become increasingly clear that this group of viruses, including poliovirus, CVB3, and EV-A71, can also spread between cells in a non-lytic fashion via extracellular microvesicles (EMVs), such as exosomes (Bird et al., 2014; Robinson et al., 2014; Chen et al., 2015; Too et al., 2016). In doing so, EVs acquire a membrane shield against host immune detection. It is thus conceivable that EV-induced secretion of EMVs represents a mechanism for the transmission of misfolded proteins (e.g., misfolded SOD1 and TDP-43) within the CNS during chronic infection.

\section{CONCLUSION}

SALS is an idiopathic, fatal neurodegenerative disorder of the motor neuron system, with no effective treatment to date. An EV etiology of SALS has been proposed for decades; however, human viral interrogation studies show conflicting results. Emerging evidence has revealed that $\mathrm{EV}$ infection induces signature molecular features of ALS. This evidence, along with the earlier findings that EVs can establish a persistent infection in the CNS, suggests that chronic and persistent EV infection might be a causal/risk factor for ALS. Further investigations are needed 
to firmly establish the relationship, for example, by assessing whether subclinical EV infection can promote early onset and progression of ALS in normal mice or mice that are genetically susceptible to ALS.

Recent progress has led to a greater recognition of the significant clinical and genetic overlaps between ALS and frontotemporal dementia (FTD). In fact, $\sim 13 \%$ of ALS patients also have FTD (Brown and Al-Chalabi, 2017; Hardiman et al., 2017; van Es et al., 2017). Mutations in the same set of genes, including C9ORF72, TARDBP, FUS, TIA1 and SQSTM1/p62, that cause ALS, are also linked to FTD (Gao et al., 2017). In addition, ALS and FTD also share common neuropathological hallmarks and disease mechanisms. It is therefore reasonable to postulate that EV infection also plays a role in FTD. Finally, identification of $\mathrm{EV}$ as a novel causal/risk factor for SALS will offer a huge potential for future therapeutic interventions. During EV infection, the use of ribavirin, a general anti-RNA viral drug that is able to cross the $\mathrm{BBB}$, long after the

\section{REFERENCES}

Al-Chalabi, A., Calvo, A., Chio, A., Colville, S., Ellis, C. M., Hardiman, O., et al. (2014). Analysis of amyotrophic lateral sclerosis as a multistep process: a population-based modelling study. Lancet Neurol. 13, 1108-1113. doi: 10.1016/S1474-4422(14)70219-4

Al-Chalabi, A., van den Berg, L. H., and Veldink, J. (2017). Gene discovery in amyotrophic lateral sclerosis: implications for clinical management. Nat. Rev. Neurol. 13, 96-104. doi: 10.1038/nrneurol.2016.182

Almer, G., Guégan, C., Teismann, P., Naini, A., Rosoklija, G., Hays, A. P., et al. (2001). Increased expression of the pro-inflammatory enzyme cyclooxygenase-2 in amyotrophic lateral sclerosis. Ann. Neurol. 49, 176-185. doi: 10.1002/1531-8249(20010201)49:2<176::aid-ana37>3.3.co;2-o

Anastasina, M., Domanska, A., Palm, K., and Butcher, S. (2017). Human picornaviruses associated with neurological diseases and their neutralization by antibodies. J. Gen. Virol. 98, 1145-1158. doi: 10.1099/jgv.0.000780

Andréoletti, L., Bourlet, T., Moukassa, D., Rey, L., Hot, D., Li, Y., et al. (2000). Enteroviruses can persist with or without active viral replication in cardiac tissue of patients with end-stage ischemic or dilated cardiomyopathy. J. Infect. Dis. 182, 1222-1227. doi: 10.1086/315818

Andrews, W. D., Tuke, P. W., Al-Chalabi, A., Gaudin, P., Ijaz, S., Parton, M. J., et al. (2000). Detection of reverse transcriptase activity in the serum of patients with motor neurone disease. J. Med. Virol. 61, 527-532. doi: 10.1002/10969071(200008)61:4<527::aid-jmv17>3.0.co;2-a

Araujo, A. Q. (2015). update on neurological manifestations of HTLV-1 infection. Curr. Infect. Dis. Rep. 17:459. doi: 10.1007/s11908-014-0459-0

Berger, M. M., Kopp, N., Vital, C., Redl, B., Aymard, M., and Lina, B. (2000). Detection and cellular localization of enterovirus RNA sequences in spinal cord of patients with ALS. Neurology 54, 20-25. doi: 10.1212/wnl.54.1.20

Bird, S. W., Maynard, N. D., Covert, M. W., and Kirkegaard, K. (2014). Nonlytic viral spread enhanced by autophagy components. Proc. Natl. Acad. Sci. U S A 111, 13081-13086. doi: 10.1073/pnas.1401437111

Boeynaems, S., Bogaert, E., Van Damme, P., and Van Den Bosch, L. (2016). Inside out: the role of nucleocytoplasmic transport in ALS and FTLD. Acta Neuropathol. 132, 159-173. doi: 10.1007/s00401-016-1586-5

Bowen, L. N., Tyagi, R., Li, W., Alfahad, T., Smith, B., Wright, M., et al. (2016). $\mathrm{HIV}$-associated motor neuron disease: HERV-K activation and response to antiretroviral therapy. Neurology 87, 1756-1762. doi: 10.1212/WNL. 0000000000003258

Brown, R. H. Jr., and Al-Chalabi, A. (2017). Amyotrophic lateral sclerosis. N. Engl. J. Med. 377:1602. doi: 10.1056/NEJMc1710379

Chapman, N. M., and Kim, K. S. (2008). Persistent coxsackievirus infection: enterovirus persistence in chronic myocarditis and dilated cardiomyopathy. Curr. Top. Microbiol. Immunol. 323, 275-292. doi: 10.1007/978-3-540-755 46-3_13 end of the acute infection can greatly reduce viral-mediated neuropathology (Ruller et al., 2012). Moreover, pleconaril, an anti-picornavirus drug with demonstrated efficacy against many EVs, including EV-D68 (Liu et al., 2015), has been shown to cross the $\mathrm{BBB}$ and limit multiple species of $\mathrm{EV}$ infection within the CNS (Schmidtke et al., 2009). Thus, it is anticipated that anti-viral therapy offers new hope for the treatment of ALS.

\section{AUTHOR CONTRIBUTIONS}

All authors contributed to the writing and discussion of this review article.

\section{ACKNOWLEDGMENTS}

This work was supported by the ALS Society of Canada.

Chen, Y. H., Du, W., Hagemeijer, M. C., Takvorian, P. M., Pau, C., Cali, A., et al. (2015). Phosphatidylserine vesicles enable efficient en bloc transmission of enteroviruses. Cell 160, 619-630. doi: 10.1016/j.cell.2015.01.032

Chen, C. S., Yao, Y. C., Lin, S. C., Lee, Y. P., Wang, Y. F., Wang, J. R., et al. (2007). Retrograde axonal transport: a major transmission route of enterovirus 71 in mice. J. Virol. 81, 8996-9003. doi: 10.1128/jvi.00236-07

Chia, R., Chiò, A., and Traynor, B. J. (2018). Novel genes associated with amyotrophic lateral sclerosis: diagnostic and clinical implications. Lancet Neurol. 17, 94-102. doi: 10.1016/S1474-4422(17)30401-5

Cipolat Mis, M. S., Brajkovic, S., Frattini, E., Di Fonzo, A., and Corti, S. (2016). Autophagy in motor neuron disease: key pathogenetic mechanisms and therapeutic targets. Mol. Cell. Neurosci. 72, 84-90. doi: 10.1016/j.mcn.2016. 01.012

Corona, A. K., Saulsbery, H. M., Corona Velazquez, A. F., and Jackson, W. T. (in press). Enteroviruses remodel autophagic trafficking through regulation of host SNARE proteins to promote virus replication and cell exit. Cell Rep.

Couderc, T., Guivel-Benhassine, F., Calaora, V., Gosselin, A. S., and Blondel, B. (2002). An ex vivo murine model to study poliovirus-induced apoptosis in nerve cells. J. Gen. Virol. 83, 1925-1930. doi: 10.1099/0022-1317-83-8-1925

Dalakas, M. C. (1995). Pathogenetic mechanisms of post-polio syndrome: morphological, electrophysiological, virological, and immunological correlations. Ann. N Y Acad. Sci. 753, 167-185. doi: 10.1111/j.1749-6632. 1995.tb27543.x

DeJesus-Hernandez, M., Mackenzie, I. R., Boeve, B. F., Boxer, A. L., Baker, M., Rutherford, N. J., et al. (2011). Expanded GGGGCC hexanucleotide repeat in noncoding region of C9ORF72 causes chromosome 9p-linked FTD and ALS. Neuron 72, 245-256. doi: 10.1016/j.neuron.2011.09.011

Douville, R., Liu, J., Rothstein, J., and Nath, A. (2011). Identification of active loci of a human endogenous retrovirus in neurons of patients with amyotrophic lateral sclerosis. Ann. Neurol. 69, 141-151. doi: 10.1002/ana.22149

Feuer, R., Mena, I., Pagarigan, R., Slifka, M. K., and Whitton, J. L. (2002). Cell cycle status affects coxsackievirus replication, persistence, and reactivation in vitro. J. Virol. 76, 4430-4440. doi: 10.1128/jvi.76.9.4430-4440.2002

Feuer, R., Pagarigan, R. R., Harkins, S., Liu, F., Hunziker, I. P., and Whitton, J. L. (2005). Coxsackievirus targets proliferating neuronal progenitor cells in the neonatal CNS. J. Neurosci. 25, 2434-2444. doi: 10.1523/JNEUROSCI.4517 $-04.2005$

Feuer, R., Ruller, C. M., An, N., Tabor-Godwin, J. M., Rhoades, R. E., Maciejewski, S., et al. (2009). Viral persistence and chronic immunopathology in the adult central nervous system following Coxsackievirus infection during the neonatal period. J. Virol. 83, 9356-9369. doi: 10.1128/JVI.02382-07

Freibaum, B. D., Lu, Y., Lopez-Gonzalez, R., Kim, N. C., Almeida, S., Lee, K. H., et al. (2015). GGGGCC repeat expansion in C9orf72 compromises nucleocytoplasmic transport. Nature 525, 129-133. doi: 10.1038/nature 14974 
Freistadt, M. S., and Eberle, K. E. (1996). Correlation between poliovirus type 1 Mahoney replication in blood cells and neurovirulence. J. Virol. 70, 6486-6492.

Fung, G., Shi, J., Deng, H., Hou, J., Wang, C., Hong, A., et al. (2015). Cytoplasmic translocation, aggregation and cleavage of TDP-43 by enteroviral proteases modulate viral pathogenesis. Cell Death Differ. 22, 2087-2097. doi: $10.1038 / \mathrm{cdd} .2015 .58$

Gao, F. B., Almeida, S., and Lopez-Gonzalez, R. (2017). Dysregulated molecular pathways in amyotrophic lateral sclerosis-frontotemporal dementia spectrum disorder. EMBO J. 36, 2931-2950. doi: 10.15252/embj.201797568

Giraud, P., Beaulieux, F., Ono, S., Shimizu, N., Chazot, G., and Lina, B. (2001). Detection of enteroviral sequences from frozen spinal cord samples of Japanese ALS patients. Neurology 56, 1777-1778. doi: 10.1212/WNL.56.12.1777

Grad, L. I., Fernando, S. M., and Cashman, N. R. (2015). From molecule to molecule and cell to cell: prion-like mechanisms in amyotrophic lateral sclerosis. Neurobiol. Dis. 77, 257-265. doi: 10.1016/j.nbd.2015.02.009

Grad, L. I., Pokrishevsky, E., Silverman, J. M., and Cashman, N. R. (2014a). Exosome-dependent and independent mechanisms are involved in prion-like transmission of propagated $\mathrm{Cu} / \mathrm{Zn}$ superoxide dismutase misfolding. Prion 8 , 331-335. doi: 10.4161/19336896.2014.983398

Grad, L. I., Yerbury, J. J., Turner, B. J., Guest, W. C., Pokrishevsky, E., O’Neill, M. A., et al. (2014b). Intercellular propagated misfolding of wild-type $\mathrm{Cu} / \mathrm{Zn}$ superoxide dismutase occurs via exosome-dependent and -independent mechanisms. Proc. Natl. Acad. Sci. U S A 111, 3620-3625. doi: 10.1073/pnas. 1312245111

Greninger, A. L., Naccache, S. N., Messacar, K., Clayton, A., Yu, G., Somasekar, S., et al. (2015). A novel outbreak enterovirus D68 strain associated with acute flaccid myelitis cases in the USA (2012-14): a retrospective cohort study. Lancet Infect. Dis. 15, 671-682. doi: 10.1016/s1473-3099(15)70093-9

Gromeier, M., and Wimmer, E. (1998). Mechanism of injury-provoked poliomyelitis. J. Virol. 72, 5056-5060.

Gustin, K. E., and Sarnow, P. (2001). Effects of poliovirus infection on nucleocytoplasmic trafficking and nuclear pore complex composition. EMBO J. 20, 240-249. doi: 10.1093/emboj/20.1.240

Han, J., Ma, X. J., Wan, J. F., Liu, Y. H., Han, Y. L., Chen, C., et al. (2010). Long persistence of EV71 specific nucleotides in respiratory and feces samples of the patients with Hand-Foot-Mouth Disease after recovery. BMC Infect. Dis. 10:178. doi: 10.1186/1471-2334-10-178

Hardiman, O., Al-Chalabi, A., Chio, A., Corr, E. M., Logroscino, G., Robberecht, W., et al. (2017). Amyotrophic lateral sclerosis. Nat. Rev. Dis. Primers 3:17085. doi: 10.1038/nrdp.2017.85

Hixon, A. M., Yu, G., Leser, J. S., Yagi, S., Clarke, P., Chiu, C. Y., et al. (2017). A mouse model of paralytic myelitis caused by enterovirus D68. PLoS Pathog. 13:e1006199. doi: 10.1371/journal.ppat.1006199

Huang, H. I., Lin, J. Y., Chen, H. H., Yeh, S. B., Kuo, R. L., Weng, K. F., et al. (2014). Enterovirus 71 infects brain-derived neural progenitor cells. Virology 468-470, 592-600. doi: 10.1016/j.virol.2014.09.017

Huang, H. I., and Shih, S. R. (2015). Neurotropic enterovirus infections in the central nervous system. Viruses 7, 6051-6066. doi: 10.3390/v7112920

Ito, D., Hatano, M., and Suzuki, N. (2017). RNA binding proteins and the pathological cascade in ALS/FTD neurodegeneration. Sci. Transl. Med. 9:eaah5436. doi: 10.1126/scitranslmed.aah5436

Jovicic, A., Mertens, J., Boeynaems, S., Bogaert, E., Chai, N., Yamada, S. B., et al. (2015). Modifiers of C9orf72 dipeptide repeat toxicity connect nucleocytoplasmic transport defects to FTD/ALS. Nat. Neurosci. 18, 1226-1229. doi: 10.1038/nn.4085

Julien, J., Leparc-Goffart, I., Lina, B., Fuchs, F., Foray, S., Janatova, I., et al. (1999). Postpolio syndrome: poliovirus persistence is involved in the pathogenesis. J. Neurol. 246, 472-476. doi: 10.1007/s004150050386

Katyal, N., and Govindarajan, R. (2017). Shortcomings in the current amyotrophic lateral sclerosis trials and potential solutions for improvement. Front. Neurol. 8:521. doi: 10.3389/fneur.2017.00521

Lee, E. B., Lee, V. M., and Trojanowski, J. Q. (2012). Gains or losses: molecular mechanisms of TDP43-mediated neurodegeneration. Nat. Rev. Neurosci. 13, 38-50. doi: 10.1038/nrn3121

Li, W., Lee, M. H., Henderson, L., Tyagi, R., Bachani, M., Steiner, J., et al. (2015). Human endogenous retrovirus-K contributes to motor neuron disease. Sci. Transl. Med. 7:307ra153. doi: 10.1126/scitranslmed.aac8201
Limongi, D., and Baldelli, S. (2016). Redox imbalance and viral infections in neurodegenerative diseases. Oxid. Med. Cell. Longev. 2016:6547248. doi: $10.1155 / 2016 / 6547248$

Lin, Y. W., Chang, K. C., Kao, C. M., Chang, S. P., Tung, Y. Y., and Chen, S. H. (2009a). Lymphocyte and antibody responses reduce enterovirus 71 lethality in mice by decreasing tissue viral loads. J. Virol. 83, 6477-6483. doi: 10.1128/JVI. 00434-09

Lin, Y. W., Wang, S. W., Tung, Y. Y., and Chen, S. H. (2009b). Enterovirus 71 infection of human dendritic cells. Exp. Biol. Med. (Maywood) 234, 1166-1173. doi: 10.3181/0903-RM-116

Lin, T. Y., Hsia, S. H., Huang, Y. C., Wu, C. T., and Chang, L. Y. (2003). Proinflammatory cytokine reactions in enterovirus 71 infections of the central nervous system. Clin. Infect. Dis. 36, 269-274. doi: 10.1086/345905

Lin, J. Y., Shih, S. R., Pan, M., Li, C., Lue, C. F., Stollar, V., et al. (2009). hnRNP Al interacts with the $5^{\prime}$ untranslated regions of enterovirus 71 and Sindbis virus RNA and is required for viral replication. J. Virol. 83, 6106-6114. doi: 10.1128/jvi.02476-08

Liu, Y., Sheng, J., Fokine, A., Meng, G., Shin, W. H., Long, F., et al. (2015). Structure and inhibition of EV-D68, a virus that causes respiratory illness in children. Science 347, 71-74. doi: 10.1126/science.1261962

Lloyd, R. E. (2015). Nuclear proteins hijacked by mammalian cytoplasmic plus strand RNA viruses. Virology 479-480, 457-474. doi: 10.1016/j.virol.2015. 03.001

MacGowan, D. J., Scelsa, S. N., Imperato, T. E., Liu, K. N., Baron, P., and Polsky, B. (2007). A controlled study of reverse transcriptase in serum and CSF of HIV-negative patients with ALS. Neurology 68, 1944-1946. doi: 10.1212/01. WNL.0000263188.77797.99

Mackenzie, I. R., Bigio, E. H., Ince, P. G., Geser, F., Neumann, M., Cairns, N. J., et al. (2007). Pathological TDP-43 distinguishes sporadic amyotrophic lateral sclerosis from amyotrophic lateral sclerosis with SOD1 mutations. Ann. Neurol. 61, 427-434. doi: 10.1002/ana.21147

Mackenzie, I. R., Nicholson, A. M., Sarkar, M., Messing, J., Purice, M. D., Pottier, C., et al. (2017). TIAl mutations in amyotrophic lateral sclerosis and frontotemporal dementia promote phase separation and alter stress granule dynamics. Neuron 95, 808.e9-816.e9. doi: 10.1016/j.neuron.2017. 07.025

Martinez, A., Palomo Ruiz, M. D., Perez, D. I., and Gil, C. (2017). Drugs in clinical development for the treatment of amyotrophic lateral sclerosis. Expert Opin. Investig. Drugs 26, 403-414. doi: 10.1080/13543784.2017.1302426

McCormick, A. L., Brown, R. H. Jr., Cudkowicz, M. E., Al-Chalabi, A., and Garson, J. A. (2008). Quantification of reverse transcriptase in ALS and elimination of a novel retroviral candidate. Neurology 70, 278-283. doi: 10.1212/01.WNL.0000297552.13219.b4

Messacar, K., Schreiner, T. L., Van Haren, K., Yang, M., Glaser, C. A., Tyler, K. L., et al. (2016). Acute flaccid myelitis: a clinical review of US cases 2012-2015. Ann. Neurol. 80, 326-338. doi: 10.1002/ana.24730

Mizutani, T., Ishizaka, A., and Nihei, C. (2016). Transferrin receptor 1 facilitates poliovirus permeation of mouse brain capillary endothelial cells. J. Biol. Chem. 291, 2829-2836. doi: 10.1074/jbc.M115.690941

Mohamud, Y., Shi, J., Qu, J., Poon, T. X., Xue, Y. C., Deng, H., et al. (in press). Enteroviral infection inhibits autophagic flux via disruption of the SNARE complex to enhance viral replication. Cell Rep.

Nagata, N., Iwasaki, T., Ami, Y., Tano, Y., Harashima, A., Suzaki, Y., et al. (2004). Differential localization of neurons susceptible to enterovirus 71 and poliovirus type 1 in the central nervous system of cynomolgus monkeys after intravenous inoculation. J. Gen. Virol. 85, 2981-2989. doi: 10.1099/vir.0. 79883-0

Neumann, M., Sampathu, D. M., Kwong, L. K., Truax, A. C., Micsenyi, M. C. Chou, T. T., et al. (2006). Ubiquitinated TDP-43 in frontotemporal lobar degeneration and amyotrophic lateral sclerosis. Science 314, 130-133. doi: $10.1126 /$ science. 1134108

Nix, W. A., Berger, M. M., Oberste, M. S., Brooks, B. R., McKenna-Yasek, D. M., Brown, R. H. Jr., et al. (2004). Failure to detect enterovirus in the spinal cord of ALS patients using a sensitive RT-PCR method. Neurology 62, 1372-1377. doi: 10.1212/01.WNL.0000123258.86752.51

Oikarinen, M., Tauriainen, S., Oikarinen, S., Honkanen, T., Collin, P., Rantala, I., et al. (2012). Type 1 diabetes is associated with enterovirus infection in gut mucosa. Diabetes 61, 687-691. doi: 10.2337/db11-1157 
Ong, K. C., and Wong, K. T. (2015). Understanding enterovirus 71 neuropathogenesis and its impact on other neurotropic enteroviruses. Brain Pathol. 25, 614-624. doi: 10.1111/bpa.12279

Park, N., Katikaneni, P., Skern, T., and Gustin, K. E. (2008). Differential targeting of nuclear pore complex proteins in poliovirus-infected cells. J. Virol. 82, 1647-1655. doi: 10.1128/jvi.01670-07

Park, N., Schweers, N. J., and Gustin, K. E. (2015). Selective removal of FG repeat domains from the nuclear pore complex by enterovirus 2A(pro). J. Virol. 89, 11069-11079. doi: 10.1128/JVI.00956-15

Ravits, J. (2005). Sporadic amyotrophic lateral sclerosis: a hypothesis of persistent (non-lytic) enteroviral infection. Amyotroph. Lateral Scler. Other Motor Neuron Disord. 6, 77-87. doi: 10.1080/14660820510027026

Renton, A. E., Majounie, E., Waite, A., Simón-Sánchez, J., Rollinson, S., Gibbs, J. R., et al. (2011). A hexanucleotide repeat expansion in C9ORF72 is the cause of chromosome 9p21-linked ALS-FTD. Neuron 72, 257-268. doi: 10.1016/j.neuron.2011.09.010

Rhoades, R. E., Tabor-Godwin, J. M., Tsueng, G., and Feuer, R. (2011). Enterovirus infections of the central nervous system. Virology 411, 288-305. doi: 10.1016/j. virol.2010.12.014

Robertson, J., Beaulieu, J. M., Doroudchi, M. M., Durham, H. D., Julien, J. P., and Mushynski, W. E. (2001). Apoptotic death of neurons exhibiting peripherin aggregates is mediated by the proinflammatory cytokine tumor necrosis factor- $\alpha$. J. Cell Biol. 155, 217-226. doi: 10.1083/jcb.200107058

Robinson, S. M., Tsueng, G., Sin, J., Mangale, V., Rahawi, S., McIntyre, L. L., et al. (2014). Coxsackievirus B exits the host cell in shed microvesicles displaying autophagosomal markers. PLoS Pathog. 10:e1004045. doi: 10.1371/journal. ppat. 1004045

Ruller, C. M., Tabor-Godwin, J. M., Van Deren, D. A. Jr., Robinson, S. M., Maciejewski, S., Gluhm, S., et al. (2012). Neural stem cell depletion and CNS developmental defects after enteroviral infection. Am. J. Pathol. 180, 1107-1120. doi: 10.1016/j.ajpath.2011.11.016

Schmidtke, M., Wutzler, P., Zieger, R., Riabova, O. B., and Makarov, V. A. (2009). New pleconaril and [(biphenyloxy)propyl]isoxazole derivatives with substitutions in the central ring exhibit antiviral activity against pleconarilresistant coxsackievirus B3. Antiviral Res. 81, 56-63. doi: 10.1016/j.antiviral. 2008.09.002

Sekizawa, T., Openshaw, H., Ohbo, K., Sugamura, K., Itoyama, Y., and Niland, J. C. (1998). Cerebrospinal fluid interleukin 6 in amyotrophic lateral sclerosis: immunological parameter and comparison with inflammatory and non-inflammatory central nervous system diseases. J. Neurol. Sci. 154, 194-199. doi: 10.1016/s0022-510x(97)00228-1

Shaid, S., Brandts, C. H., Serve, H., and Dikic, I. (2013). Ubiquitination and selective autophagy. Cell Death Differ. 20, 21-30. doi: 10.1038/cdd. 2012.72

Shi, J., Fung, G., Piesik, P., Zhang, J., and Luo, H. (2014). Dominant-negative function of the C-terminal fragments of NBR1 and SQSTM1 generated during enteroviral infection. Cell Death Differ. 21, 1432-1441. doi: 10.1038/cdd. 2014.58

Shi, J., and Luo, H. (2012). Interplay between the cellular autophagy machinery and positive-stranded RNA viruses. Acta Biochim. Biophys. Sin. (Shanghai) 44, 375-384. doi: 10.1093/abbs/gms010

Shi, J., Wong, J., Piesik, P., Fung, G., Zhang, J., Jagdeo, J., et al. (2013). Cleavage of sequestosome 1/p62 by an enteroviral protease results in disrupted selective autophagy and impaired NFKB signaling. Autophagy 9, 1591-1603. doi: 10.4161/auto.26059

Steele, A. J., Al-Chalabi, A., Ferrante, K., Cudkowicz, M. E., Brown, R. H. Jr., and Garson, J. A. (2005). Detection of serum reverse transcriptase activity in patients with ALS and unaffected blood relatives. Neurology 64, 454-458. doi: 10.1212/01.WNL.0000150899.76130.71

Suresh, S., Forgie, S., and Robinson, J. (2018). Non-polio Enterovirus detection with acute flaccid paralysis: a systematic review. J. Med. Virol. 90, 3-7. doi: 10.1002/jmv.24933

Swanson, N. R., Fox, S. A., and Mastaglia, F. L. (1995). Search for persistent infection with poliovirus or other enteroviruses in amyotrophic lateral sclerosis-motor neurone disease. Neuromuscul. Disord. 5, 457-465. doi: 10.1016/0960-8966(95)00002-5

Tabor-Godwin, J. M., Ruller, C. M., Bagalso, N., An, N., Pagarigan, R. R., Harkins, S., et al. (2010). A novel population of myeloid cells responding to coxsackievirus infection assists in the dissemination of virus within the neonatal CNS. J. Neurosci. 30, 8676-8691. doi: 10.1523/JNEUROSCI.186010.2010

Tolbert, M., Morgan, C. E., Pollum, M., Crespo-Hernández, C. E., Li, M. L., Brewer, G., et al. (2017). HnRNP A1 alters the structure of a conserved enterovirus IRES domain to stimulate viral translation. J. Mol. Biol. 429, 2841-2858. doi: 10.1016/j.jmb.2017.06.007

Too, I. H., Yeo, H., Sessions, O. M., Yan, B., Libau, E. A., Howe, J. L., et al. (2016). Enterovirus 71 infection of motor neuron-like NSC-34 cells undergoes a non-lytic exit pathway. Sci. Rep. 6:36983. doi: 10.1038/srep36983

Tsueng, G., Tabor-Godwin, J. M., Gopal, A., Ruller, C. M., Deline, S., An, N., et al. (2011). Coxsackievirus preferentially replicates and induces cytopathic effects in undifferentiated neural progenitor cells. J. Virol. 85, 5718-5732. doi: 10.1128/JVI.02261-10

Tung, W. H., Lee, I. T., Hsieh, H. L., and Yang, C. M. (2010). EV71 induces COX-2 expression via c-Src/PDGFR/PI3K/Akt/p42/p44 MAPK/AP-1 and NF-кB in rat brain astrocytes. J. Cell. Physiol. 224, 376-386. doi: 10.1002/jcp.22133

Tyagi, R., Li, W., Parades, D., Bianchet, M. A., and Nath, A. (2017). Inhibition of human endogenous retrovirus-K by antiretroviral drugs. Retrovirology 14:21. doi: 10.1186/s12977-017-0347-4

van Es, M. A., Hardiman, O., Chio, A., Al-Chalabi, A., Pasterkamp, R. J., Veldink, J. H., et al. (2017). Amyotrophic lateral sclerosis. Lancet 390, 2084-2098. doi: 10.1016/S0140-6736(17)31287-4

Vandenberghe, N., Leveque, N., Corcia, P., Brunaud-Danel, V., SalortCampana, E., Besson, G., et al. (2010). Cerebrospinal fluid detection of enterovirus genome in ALS: a study of 242 patients and 354 controls. Amyotroph. Lateral. Scler. 11, 277-282. doi: 10.3109/17482960903262083

Verma, A., and Berger, J. R. (2006). ALS syndrome in patients with HIV-1 infection. J. Neurol. Sci. 240, 59-64. doi: 10.1016/j.jns.2005.09.005

Walker, M. P., Schlaberg, R., Hays, A. P., Bowser, R., and Lipkin, W. I. (2001). Absence of echovirus sequences in brain and spinal cord of amyotrophic lateral sclerosis patients. Ann. Neurol. 49, 249-253. doi: 10.1002/15318249(20010201)49:2<249::aid-ana46>3.3.co;2-v

Woodall, C. J., Riding, M. H., Graham, D. I., and Clements, G. B. (1994). Sequences specific for enterovirus detected in spinal cord from patients with motor neurone disease. BMJ 308, 1541-1543. doi: 10.1136/bmj.308.6943.1541

Xiao, S., Macnair, L., McGoldrick, P., McKeever, P. M., McLean, J. R., Zhang, M., et al. (2015). Isoform-specific antibodies reveal distinct subcellular localizations of C9orf72 in amyotrophic lateral sclerosis. Ann. Neurol. 78, 568-583. doi: 10.1002/ana.24469

Yang, W. X., Terasaki, T., Shiroki, K., Ohka, S., Aoki, J., Tanabe, S., et al. (1997). Efficient delivery of circulating poliovirus to the central nervous system independently of poliovirus receptor. Virology 229, 421-428. doi: 10.1006/viro. 1997.8450

Yarbrough, M. L., Mata, M. A., Sakthivel, R., and Fontoura, B. M. (2014). Viral subversion of nucleocytoplasmic trafficking. Traffic 15, 127-140. doi: $10.1111 /$ tra. 12137

Yu, B., and Pamphlett, R. (2017). Environmental insults: critical triggers for amyotrophic lateral sclerosis. Transl. Neurodegener. 6:15. doi: 10.1186/s40035017-0087-3

Zhang, K., Donnelly, C. J., Haeusler, A. R., Grima, J. C., Machamer, J. B., Steinwald, P., et al. (2015). The C9orf72 repeat expansion disrupts nucleocytoplasmic transport. Nature 525, 56-61. doi: 10.1038/nature14973

Zhang, X., Zheng, Z., Shu, B., Liu, X., Zhang, Z., Liu, Y., et al. (2013). Human astrocytic cells support persistent coxsackievirus B3 infection. J. Virol. 87, 12407-12421. doi: 10.1128/JVI.02090-13

Conflict of Interest Statement: The authors declare that the research was conducted in the absence of any commercial or financial relationships that could be construed as a potential conflict of interest.

Copyright (c) 2018 Xue, Feuer, Cashman and Luo. This is an open-access article distributed under the terms of the Creative Commons Attribution License (CC BY). The use, distribution or reproduction in other forums is permitted, provided the original author(s) and the copyright owner are credited and that the original publication in this journal is cited, in accordance with accepted academic practice. No use, distribution or reproduction is permitted which does not comply with these terms. 